\title{
Clinical features and outcomes of COVID-19 patients with benign prostatic hyperplasia in aging male: A retrospective study of 18
}

\section{cases}

Ramazan Topaktas ${ }^{1}$, Emre Tokuç², Musab Ali Kutluhan ${ }^{3}$, Mehmet Akyüz ${ }^{4}$, Emre

Karabay $^{4}$, and Selahattin Çalıskan ${ }^{5}$

${ }^{1}$ Istanbul Haydarpasa Numune Training and Research Hospital

${ }^{2}$ Sultanbeyli State Hospital

${ }^{3}$ Fatih Sultan Mehmet Training and Research Hospital

${ }^{4}$ Istsanbul Haydarpasa Numune Training and Research Hospital

${ }^{5}$ Reyap Special Hospital

April 28, 2020

\begin{abstract}
Aim: A worldwide pandemic of coronavirus disease 2019 (COVID-19) which emerged in China in December 2019 affects the world very seriously. We aimed to evaluate the benign prostatic hyperplasia (BPH) patients who were admitted and treated to our hospital due to COVID-19. Methods: Between March 18, 2020 and April 5, 2020 , 18 patients admitted with COVID-19 who has BPH and are using medication for this were included in the study and analyzed retrospectively. Diagnosis was confirmed by COVID-19 nucleic acid test by sampling sputum or nasopharyngeal swab. Standard COVID-19 treatment protocol determined by our Ministry of Health was applied to all patients according to their risk groups. Epidemiological, clinical, radiological features, additional diseases, laboratory tests, complications and outcome data of all patients were recorded. Results: Mean age of patients was 59.6 (range: 56-73). As the mode of transmission, 10 (55.5\%) of patients were infected in hospital, 5 (27.7\%) patients had a relative with COVID-19 and $3(16,6 \%)$ was unknown. During follow-up, $2(11.1 \%)$ patients were transferred to intensive care unit (ICU). One of these patients dramatically progressed and died. Patients who survived and were not transferred to ICU had lesser comorbidities and were relatively young. Mean duration of hospitalization was 14.2 days (range 12-19). Conclusion: We think that COVID-19 patients with BPH had a low mortality rate and did not have a poor prognosis in this patient group. It is crucial to take comprehensive preventive measures to control COVID-19 transmission via hospital route.
\end{abstract}

Title: Clinical features and outcomes of COVID-19 patients with benign prostatic hyperplasia in aging male: A retrospective study of 18 cases

Aim : A worldwide pandemic of coronavirus disease 2019 (COVID-19) which emerged in China in December 2019 affects the world very seriously. We aimed to evaluate the benign prostatic hyperplasia (BPH) patients who were admitted and treated to our hospital due to COVID-19.

Methods : Between March 18, 2020 and April 5, 2020, 18 patients admitted with COVID-19 who has BPH and are using medication for this were included in the study and analyzed retrospectively. Diagnosis was confirmed by COVID-19 nucleic acid test by sampling sputum or nasopharyngeal swab. Standard COVID-19 treatment protocol determined by our Ministry of Health was applied to all patients according to their risk groups. Epidemiological, clinical, radiological features, additional diseases, laboratory tests, complications and outcome data of all patients were recorded. 
Results : Mean age of patients was 59.6 (range: 56-73). As the mode of transmission, $10(55.5 \%)$ of patients were infected in hospital, $5(27.7 \%)$ patients had a relative with COVID-19 and $3(16,6 \%)$ was unknown. During follow-up, 2 (11.1\%) patients were transferred to intensive care unit (ICU). One of these patients dramatically progressed and died. Patients who survived and were not transferred to ICU had lesser comorbidities and were relatively young. Mean duration of hospitalization was 14.2 days (range 12-19).

Conclusion : We think that COVID-19 patients with BPH had a low mortality rate and did not have a poor prognosis in this patient group. It is crucial to take comprehensive preventive measures to control COVID-19 transmission via hospital route.

Keywords: Benign prostatic hyperplasia; COVID-19; Nosocomial infection; Outcome; Pandemic

What's known

* Risk management is essential for the management of

acute coronary syndrome.

* The GRACE and CRUSADE scores are amongst the most

widely used tools to estimate risk of death or major bleeding.

What's new

* The GRACE score has good prediction capability for

in-hospital death in patients with acute coronary syn-

drome, and is superior to CRUSADE.

*? It?is?also?better?than?CRUSADE?to?predict?major?bleed-

ing, although the latter score was specifically developed

for bleeding

\section{What's known}

- Coronavirus 2019 disease mostly affects the elderly, men and those with comorbid disease.

- Both BPH and COVID-19disease frequently affect older men.

- The course of COVID-19 disease in BPH patients is not known and there is no study about this topic.

\section{What's new}

- This is the first study on COVID-19 course in BPH patients in the literature.

- We think that a low mortality rate in COVID-19 patients who are known to have BPH.

\section{INTRODUCTION}

In late December 2019, a group of patients were identified with pneumonia with unknown aetiology in Wuhan, Hubei, China, and eventually, severe acute respiratory syndrome - coronavirus 2 (SARS-CoV 2) was identified by lower respiratory tract samples. ${ }^{1}$ Coronavirus disease 2019 (COVID-2019) outbreak, which infected over 1.5 million people and caused more than 92000 deaths, especially affects the elderly with comorbid diseases and male population. ${ }^{2,3}$ Symptoms of COVID-19 infection range from asymptomatic disease to acute respiratory infection and can be potentially fatal in the elderly, over 65 years of age, and those with comorbidity. Transmission routes involve droplets and close contact. Diagnosis is made by reverse transcription polymerase chain reaction (RT-PCR) of the sputum, nasopharyngeal swab or other samples.

Benign prostatic hyperplasia (BPH) is also a worldwide disease directly related to aging, and is especially common among older men. With transitioning to an aging society, the incidence and prevalence of symptomatic BPH increased rapidly. ${ }^{4}$ Prevalence of $\mathrm{BPH}$ is about $25.3 \%$ among men aged $40-79$, and more than $50 \%$ among men aged 60 and over. ${ }^{5}$ Considering that COVID-19 pandemic affecting older and male patients 
more, it is obvious that the elderly male population with BPH will be seriously affected by this pandemic. According to our literature review, there are no studies examining the clinical features, course and outcomes of COVID-19 disease in patients diagnosed with BPH.

In our study, we aimed to present our clinical and laboratory experiences related to patients diagnosed with $\mathrm{BPH}$, also known to be followed up and treated in our hospital due to COVID-19. We also aimed to contribute to the information about COVID-19 which is an urgent need.

\section{PATIENTS AND METHODS}

Eighteen male patients who were diagnosed with COVID-19 in our service between March 18, 2020 and April 5, 2020, and who had previously known BPH and who used alpha-blockers and/or five alpha-reductase inhibitors were included in the study. The study was conducted in accordance with the Declaration of Helsinki and approved by the local hospital manager. Oral consent was obtained from patients or relatives. Patients whose information could not be reached through their medical record, who had previous prostate surgery, whose prostate specific antigen (PSA) value was higher than $4 \mathrm{ng} / \mathrm{mL}$ and who never consulted an urology physician despite having complaints of lower urinary system were excluded from the study. All patients included in the study had laboratory-confirmed SARS-CoV-2 infection via sputum or nasopharyngeal swab samples evaluated through SARS-CoV2 nucleic acid RT-PCR. Whether the patient had BPH or not was investigated through the patient information system (Health Information System). A detailed history of all patients was taken and it was found that alpha blockers and/or five alpha-reductase inhibitors were prescribed by the urologist. Our hospitals are reference pandemic centers in Istanbul where there are the most SARSCoV-2 cases in our country. Clinical, radiological data, underlying comorbidities, laboratory findings during hospitalization, treatment, complications and outcomes data were collected and analyzed retrospectively. Epidemiological data such as age, body mass index (BMI), exposure to source of transmission, incubation period (defined as the time from exposure to source of transmission to onset of symptom), and familial cluster were collected. Clinical symptoms and comorbid diseases were questioned and recorded. In our clinic, standard treatments were applied and recorded according to the risk group, which was frequently revised according to current data, together with the scientific committee of our Ministry of Health. All possible complications (lymphopenia, hypoxemia, shock, acute respiratory distress syndrome (ARDS), arrhythmia, acute kidney injury, acute liver injury and acute cardiac injury) were recorded during their stay in the hospital.

\section{Statistical Analysis}

Quantitative data were expressed as mean with range and categorical variables were reported as numbers with percentages (\%). For laboratory results, we also assessed whether or not measurements fell within the normal range. SPSS v 26.0 (IBM, NY, USA) was used for all analysis.

\section{RESULTS}

Mean age of the patients was 59.6 (range, 56 to 73). Demographic data, details of comorbid diseases and complications are shown in Table 1. Route of transmission was determined as through hospital to a total of $10(55.5 \%)$ patients (hospital employee, during admission to the outpatient clinic or during a patient visit), while $5(27.7 \%)$ patients had COVID-19 patients diagnosed in their family. Most common signs and symptoms when applying to the hospital was fever (n: 15, 83.3\%), fatigue (n: 14, 77.7\%), muscle and joint pain (n: 14, 77.7\%), dry cough (n: 10,55.5\%), headache and throat pain (n: 8, 44.4\%), sudden loss of taste and smell (n: 4, 22.2\%). Median time from the first symptoms to the application was 5 (range, 2-10) days.

All patients had radiological imaging of lung (thorax computed tomography (CT) and / or chest X-ray). Chest imaging examination showed that 12 patients $(66.6 \%)$ had ground-glass opacity. Figure 1 shows a thorax CT of a 59-year-old patient (hospital worker). Impaired laboratory tests was frequently observed at admission; lymphopenia (n: 12, 72.2\%), high procalcitonin (n: 8, 44.4\%), elevated lactate dehydrogenase (n: 8, 44.4\%), high C-reactive protein (CRP) (n: 7, 38.8\%), D-dimer (n: 7, 38.8\%) and Alanine aminotransferase (ALT) (n: 7, 38.8\%). All patients were isolated and treated in single rooms. All cases were symptomatic but 
uncomplicated patients with mild / moderate pneumonia. Oxygen therapy was administered to patients when saturation decreased through nasal cannulation. As medical treatment, the standard treatment algorithm (Hydroxychloroquine 2x200mg, Oseltamivir $75 \mathrm{mg} 2 \times 75 \mathrm{mg}$, Azithromycin 1x250mg) recommended by our Ministry of Health for this case group was applied. They also continued BPH treatments (alpha blockers and/or five alpha-reductase inhibitors) during their follow-up. None of the patients developed complications related to BPH during follow-up. Mean PSA value was calculated as $2.4 \mathrm{ng} / \mathrm{mL}$ (range, 0.5-3.9 ng/mL).

During follow-up and treatment process, $2(11.1 \%)$ patients were sent to the intensive care unit (ICU) due to ARDS and acute cardiac injury. One of these patients was a 73-year-old patient with multiple comorbidities (hypertension, hyperlipidemia and diabetes type 2), and severe respiratory distress developed within hours, followed by ARDS and multi-organ failure. This patient, who was intubated in ICU, passed away after 72 hours. Treatment of a total of 16 patients was performed in the service without being sent to ICU. It was determined that the patients whose treatment were completed in the service were younger and had no comorbid diseases or less, rather than the two patients who were sent to ICU. Excessive ground-glass opacity and intensive consolidation was observed in chest imagings of two patients ( 1 alive, 1 deceased) sent to ICU. Again, during the follow-up of these patients, procalcitonin, ALT, D-dimer, troponin and CRP values were higher than other patients. Mean duration of collection of sputum / nasopharyngeal swab to the first negative result was 8.4 days (range, 5-16 days). Mean duration of hospital stay was 11.9 (range, 8-17) days.

\section{DISCUSSION}

In December 2019, the first cases of pneumonia of unknown etiology were detected in Wuhan, China. A previously unknown betacoronavirus was detected in the respiratory samples of these patients [1]. World Health Organization named the virus as SARS-CoV-2, while the disease it caused was called COVID-19 and announced it as a pandemic on March 11, 2020. ${ }^{6}$ COVID-19, which is still spreading in the world, infected approximately 1 million 709 thousand people in the world as of April 11, 2020, while the total number of deaths is over 103 thousand people. As of March 1, the mortality rates of this very contagious pandemic disease were reported to be $(5.6 \%$ and $15.6 \%)$ in China and non-Chinese countries, respectively. ${ }^{7}$ The first case in our country was diagnosed in the city of Istanbul on March 11, 2020. Until April 112020 , the number of cases exceeded 47000 and 1006 people died in a period of 1 month. Mortality rate in our country is currently $2.19 \%$.

$\mathrm{BPH}$ is a major cause of lower urinary tract symptoms among aging males, affecting approximately 210 million men all over the world. ${ }^{8}$ An estimated $50 \%$ and $75 \%$ of men have histologic evidence of BPH by the age of 50 and 80 years, respectively, with approximately $50 \%$ of them having clinically considerable symptoms. ${ }^{8}$ Current studies found that nearly half of COVID-19 patients are over the age of 50 and men are more likely to be infected than women and the mortality rate in males is higher than that in females. ${ }^{9}$ Considering the frequency of BPH disease in older men, we thought it was important to investigate the course of COVID-19 disease in these BPH patients. When the literature is researched, the association and course of COVID-19 disease with many diseases have been investigated, but there are no studies on the course of the disease in BPH patients. In our study, we found a low mortality rate in COVID-19 patients who are known to have BPH and who have been diagnosed with medical treatment. We think that the rapid development of systemic complications after the onset of COVID-19 in our single patient who died, and the fact that the patient was elderly and had more than two comorbidities which led to death. All the patients receiving standard COVID-19 therapy during follow-up also continued to use alpha blockers and/or five alpha reductase inhibitors due to $\mathrm{BPH}$. None of the patients experienced negative drug interactions and complications. Less than half of patients (n: 6, 33.3\%) required low-flow oxygen therapy, and none of the patients needed invasive ventilation or extracorporeal membrane oxygenation.

Since SARS-CoV-2 is a recent virus, currently, there is no vaccine or therapeutic antibody to prevent the infection, and more time is required to develop an effective immune strategy against the pathogen. In addition to supportive treatments, medical treatments (antivirals, antibiotics, corticosteroids, hydroxychloroquine etc.) are applied. Unfortunately, a standard treatment protocol applied worldwide has unfortunately not yet been developed. Many treatment options have been tried in China and other countries and their effects on 
the virus have been published. Russell et al. ${ }^{10}$ suggested that corticosteroid therapy should not be used other than SARS-CoV-2 induced lung injury or shock therapy. In another study had identified 4 small molecular drugs (prulifloxacin, nelfinavir, bictegravir, tegobuvir) with high binding capacity with SARS-CoV-2 main protease. ${ }^{11}$ In addition, remdesivir and chloroquine have been shown to effectively inhibit SARS-CoV-2 in vitro and have been reported as potential treatments for COVID-19 as well as baricitinib. ${ }^{12,13}$ The fact that it is seen later in our country compared to other world countries has been an advantage in terms of applying the most effective treatment. The scientific committee formed by our Ministry of Health has determined treatment algorithms according to the severity of the disease by considering the current literature and updating it continuously. We applied treatment to our patients according to this algorithm in our clinic.

Conventional ways of transmission of SARS-CoV-2 are thought to be similar to other contemporary coronavirus syndromes. However, detection of SARS-CoV-2 in the gastrointestinal tract, saliva, and urine may suggest other potential portals of transmission as well. ${ }^{14}$ Transmission of COVID-19 is mainly through respiratory droplets from cough or sneeze, direct contact and aerosol transmission is also possible. Droplets can be deposited in the mouths, noses, or eyes of people nearby or be inhaled into the lungs of those in close proximity. In the light of this information, measures including strict precautions, adequate protective devices, and infection control training should be implemented for all hospital workers, especially assistants and cleaners who handle the excreta of these patients and toilet disinfection. When the transmission path in our study was examined, 10 patients were infected in hospital environment (hospital worker, during admission to the outpatient clinic as a patient or during a visit to the patient), while 5 patients were transmitted through direct contact due to a COVID-19 patient diagnosed in the patient's family. We think that these patients were infected during the period when COVID-19 was not seen commonly in hospitals and individuals did not take adequate measures and surgical masks was not used widely in our country.

COVID-19 is mainly manifested as fever, accompanied by cough, fatigue, muscular soreness, chest depression, shortness of breath, diarrhea and other symptoms. ${ }^{15}$ In accordance with the literature, fever, fatigue, muscle and joint pain, dry cough, headache and throat pain, sudden loss of taste and smell in our patients were also determined. Most of our patients had normal or decreased white blood cell count and low lymphocyte count.

This study has several limitations. Lack of a prospective, comparative study with control group and low number of cases can be listed as the main limitations. Another limitation is that follow-up times are very short. Despite all these limitations, we know that our study is the first and only study on this subject, and we think that it may be helpful in this period. Although there are deficiencies, it can be a guide for the studies to be carried out and contribute to the information about COVID-19 disease that is still spreading rapidly.

\section{CONCLUSION}

COVID-19, announced as a pandemic, is highly contagious. Hospitalized patients, visitors and hospital staff are at high risk of SARS-CoV-2 infection during the pandemic. The mortality rate of our patients with COVID-19, known to be BPH, was found to be low and we think that COVID-19 may not have a poor prognosis in this patient group. We hope that current and future research on COVID-19 can answer questions, and that all humanity will deal wisely with this crisis.

\section{ETHICAL APPROVAL}

Informed consent was obtained from all individual participants included in the study. All procedures performed in studies were in accordance with the ethical standards of the institutional and / or national research committee and with the 1964 Helsinki declaration and its later amendments or comparable ethical standards.

\section{CONFLICTS OF INTEREST}

All authors have no conflict of interest to declare

\section{REFERENCES}


1. Zhu N, Zhang D, Wang W, et al. A novel coronavirus from patients with pneumonia in china, 2019. $N$ Engl J Med. 2020;382:727-733.

2. Chen Z, Fan H, Cai J, et al. High-resolution computed tomography manifestations of COVID-19 infections in patients of different ages.Eur J Radiol . 2020;126:108972.

3. World Health Organization, Coronavirus Disease 2019 (COVID-19) Situation Report - 54, (2020) https://www.who.int/docs/default-source/coronaviruse/situationreports/20200408-sitrep-54-covid-19.pdf?sfvrsn=dcd46351 6.

4. Kim BS, Ko YH, Song PH, Kim TH, Kim KH, Kim BH. Prostatic urethral length as a predictive factor for surgical treatment of benign prostatic hyperplasia: a prospective, multiinstitutional study.Prostate Int. 2019;7:30-34.

5. Peng YH, Huang CW, Chou CY, et al. Association between asthma and risk of benign prostatic hyperplasia: a retrospective population-based study. Aging Male. 2019;11:1-8.

6. World Health Organization. Situation Report 51, 2020 https://www.who.int/docs/defaultsource/coronaviruse/situationreports/20200311-sitrep-51-covid19.pdf

7. Baud D, Qi X, Nielsen-Saines K, Musso D, Pomar L, Favre G. Real estimates of mortality following COVID-19 infection. Lancet Infect Dis. 2020. doi: 10.1016/S1473-3099(20)30195-X.

8. Hou CP, Lin YH, Chen TH, et al. Transurethral resection of the prostate achieves favorable outcomes in stroke patients with symptomatic benign prostate hyperplasia. Aging Male. 2018;21:9-16.

9. Du Y, Tu L, Zhu P, et al. Clinical features of 85 fatal cases of covid-19 from Wuhan: a retrospective observational study. Am J Respir Crit Care Med. 2020. doi: 10.1164/rccm.202003-0543OC

10. Russell CD, Millar JE, Baillie JK. Clinical evidence does not support corticosteroid treatment for 2019nCoV lung injury.Lancet. 2020;395:473-475.

11. Li Y, Zhang J, Wang N, et al. Therapeutic drugs targeting 2019-ncov main protease by high-throughput screening. bioRxiv. 2020. doi: 2020.01.28.922922.

12. Wang M, Cao R, Zhang L, et al. Remdesivir and chloroquine effectively inhibit the recently emerged novel coronavirus (2019-nCoV) in vitro. Cell Res. 2020;30:269-271.

13. Richardson P, Griffin I, Tucker C, et al. Baricitinib as potential treatment for 2019-nCoV acute respiratory disease. Lancet.2020;395:e30-e31.

14. Guan WJ, Ni ZY, Hu Y, et al. Clinical characteristics of coronavirus disease 2019 in china. $N$ Engl $J$ Med. 2020. doi: 10.1056/NEJMoa2002032.

15. Li YK, Peng S, Li LQ, et al. Clinical and transmission characteristics of covid-19 - a retrospective study of 25 cases from a single thoracic surgery department. Curr Med Sci . 2020. doi: 10.1007/s11596-020-2176-2.

TABLE 1 Summary of characteristics, complications and outcome of patients with COVID-19

\begin{tabular}{llll}
\hline Parameters & All $(\mathbf{n}, \mathbf{\%})$ & Survivors & Non-survivor \\
\hline & 18 & 17 & 1 \\
Age, years & $59,6(56$ to 73$)$ & $57,8(56$ to 70$)$ & 73 \\
BMI, $\mathrm{kg} / \mathrm{m} 2$ & $25.4(20,4-27.1)$ & $25.2(20.4-26.3)$ & 27.1 \\
$\begin{array}{l}\text { Route of } \\
\text { transmission }\end{array}$ & & \\
Familial cluster & $5,27.7 \%$ & 4 & 1 \\
Health care workers & $2,11.1 \%$ & 2 & \\
Appliance to outpatient & $8,44,4 \%$ & 8 & \\
clinic or visitor & & &
\end{tabular}




\begin{tabular}{|c|c|c|c|}
\hline Parameters & All $(n, \%)$ & Survivors & Non-survivor \\
\hline \multicolumn{4}{|l|}{ Signs and symptoms } \\
\hline Fever $(>37.5)$ & $15,83.3 \%$ & 14 & 1 \\
\hline Fatigue & $14,77.7 \%$ & 13 & 1 \\
\hline Muscle or joint ache & $14,77.7 \%$ & 13 & 1 \\
\hline Dry cough & $10,55,5 \%$ & 9 & 1 \\
\hline Headache and sore throat & $8,44.4 \%$ & 8 & \\
\hline Taste or smell loss & $4,22.2 \%$ & 4 & \\
\hline Diarrhea & $3,16.6 \%$ & 3 & \\
\hline $\begin{array}{l}\text { More than one sign or } \\
\text { symptom }\end{array}$ & $17,94,4 \%$ & 16 & 1 \\
\hline \multicolumn{4}{|l|}{ Comorbidities } \\
\hline None & $6,33,3 \%$ & & \\
\hline Smoking & 7 & 6 & 1 \\
\hline Hypertension & 4 & 3 & 1 \\
\hline Diabetes & 2 & 1 & 1 \\
\hline Cerebrovascular disease & 2 & 2 & \\
\hline Hyperlipidemia & 1 & & 1 \\
\hline Respiratory diseases & 1 & 1 & \\
\hline Cardiovascular disease & 1 & 1 & \\
\hline Malignancy & 1 & 1 & \\
\hline \multicolumn{4}{|l|}{ Complications } \\
\hline Lymphopenia & $12,72.2 \%$ & 11 & 1 \\
\hline Acute liver injury & $7,38,8 \%$ & 6 & 1 \\
\hline Acute bacterial infection & $5,27,7 \%$ & 4 & 1 \\
\hline ARDS & $1,5,5 \%$ & & 1 \\
\hline Acute cardiac injury & $1,5,5 \%$ & 1 & \\
\hline Septic shock & $1,5,5 \%$ & & 1 \\
\hline Multiple organ failure & $1,5,5 \%$ & & 1 \\
\hline \multicolumn{4}{|l|}{ Outcomes } \\
\hline Discharge & $17,94.4 \%$ & & \\
\hline Deceased & $1,5.5 \%$ & & \\
\hline ARDS & $1,5.5 \%$ & & \\
\hline Cardiac arrest & $1,5.5 \%$ & & \\
\hline Respiratory Failure & $1,5.5 \%$ & & \\
\hline
\end{tabular}

BMI: body mass index, ARDS: Acute respiratory distress syndrome

FIGURE 1 Chest computed tomography images (lung window) in the axial (A) and coronal (B) planes show peripheral ground glass-opacities and consolidation. 


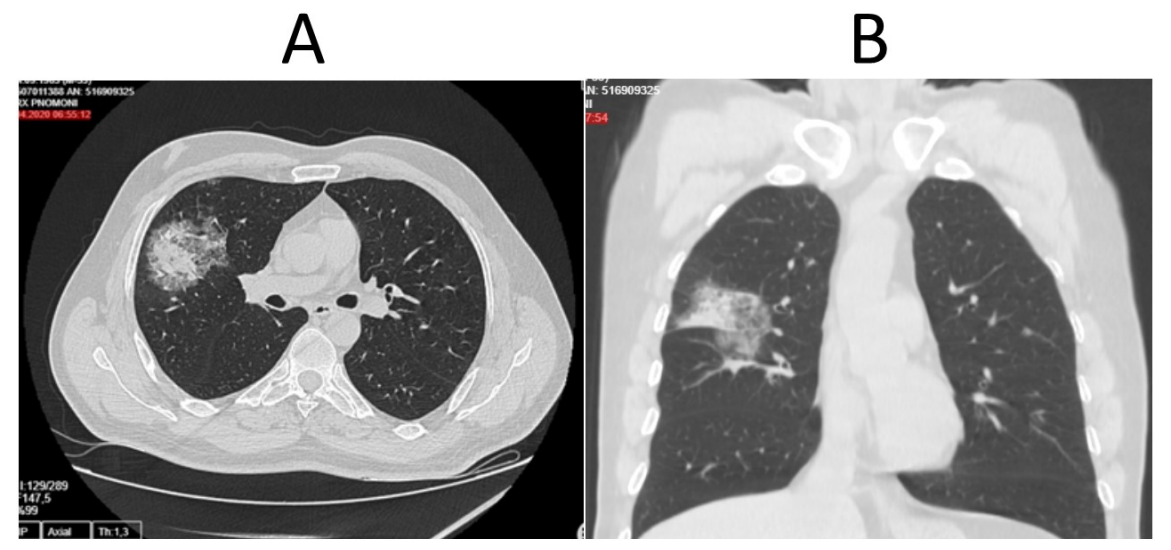

\section{Hosted file}

Table .docx available at https://authorea.com/users/313369/articles/443892-clinical-featuresand-outcomes-of-covid-19-patients-with-benign-prostatic-hyperplasia-in-aging-male-a-retrospectivestudy-of-18-cases 